\title{
Neuronal Signatures of Negative and Positive Schemas towards the Self and Others in Patients with Early Stage Schizophrenia
}

\author{
Jie Shen', Guangfan Shen', Woo-Sung Kim¹, Uyanga Tsogt', \\ Congcong Liu', Jing Sui ${ }^{2,3}$, and Young-Chul Chung ${ }^{1,4,5} \bowtie$ \\ 1Department of Psychiatry, Jeonbuk National University Medical School, Jeonju, Republic of Korea \\ ${ }^{2}$ Brainnetome Center and National Laboratory of Pattern Recognition, Institute of Automation, Chinese Academy of Sciences, Beijing, China \\ ${ }^{3}$ University of Chinese Academy of Sciences, CAS Center for Excellence in Brain Science and Intelligence Technology, \\ Chinese Academy of Sciences, Beijing, China \\ ${ }^{4}$ Department of Psychiatry, Jeonbuk National University Hospital, Jeonju, Republic of Korea \\ ${ }^{5}$ Research Institute of Clinical Medicine of Jeonbuk National University-Biomedical Research Institute of Jeonbuk National University Hospital, \\ Jeonju, Republic of Korea
}

Objective The present study investigated the functional neuroanatomy underlying negative and positive schemas towards the self and others in patients with early stage schizophrenia spectrum disorders (SSDs) using a task-based fMRI procedure.

Methods This study included 50 patients with SSDs and 52 controls. The schema-evoking task consisted of four active conditions and neutral condition. Differences in brain activation were compared between the two groups. Correlation analysis was performed between task-related activation and psychopathology.

Results The SSD patients exhibited higher activity of the left middle and inferior frontal gyri under the negative-others minus neutral contrast as well as greater activation of the left superior and middle frontal gyri and right medial superior frontal gyrus under the positive-self minus neutral and positive-others minus neutral contrasts. Under the positive-others minus neutral contrast, negative correlation was observed between activity of the right inferior parietal gyrus and right angular and total score of the Positive and Negative Syndrome Scale (PANSS), whereas positive correlation between activity of the left middle cingulate gyrus and left/right precuneus and positive-others score of the Brief Core Schema Scales (BCSS).

Conclusion The present findings suggest that the frontal brain regions of SSD patients are more sensitive to negative and positive schemas towards the self and/or others compared to those of controls.

Psychiatry Investig 2021;18(4):284-294

Key Words Negative schema, Positive schema, Schizophrenia, fMRI.

\section{INTRODUCTION}

Schizophrenia is the most representative severe mental illness. It is a very complex and heterogeneous disorder that involves synergistic interactions among various biological, psychological, and social factors to manifest its symptoms. Recently, there has been emerging interest towards the self-disorder of schizophrenia. ${ }^{1}$ Using various designs of task-based fMRI, neural correlates underlying the psychopathology related to self

Received: September 3, 2020 Revised: December 12, 2020

Accepted: December 18, 2020

$\triangle$ Correspondence: Young-Chul Chung, MD, PhD

Department of Psychiatry, Jeonbuk National University Medical School, 20 Geonji-ro, Deokjin-gu, Jeonju 54907, Republic of Korea

Tel: +82-63-250-2185, Fax: +82-63-275-3157, E-mail: chungyc@jbnu.ac.kr

(c) This is an Open Access article distributed under the terms of the Creative Commons Attribution Non-Commercial License (https://creativecommons.org/licenses/by$\mathrm{nc} / 4.0$ ) which permits unrestricted non-commercial use, distribution, and reproduction in any medium, provided the original work is properly cited. or others in schizophrenia can be investigated.

People with chronic psychosis report extreme negative evaluations of the self and others, ${ }^{2}$ and these negative schemas are thought to be formed early in life and then remain stable throughout adulthood. ${ }^{3}$ It has been suggested that sexual and physical abuse experienced during childhood contribute to the development of schemas related to the "hazard" theme, whereas childhood neglect may lead to the development of schemas related to themes of "loss" and "insignificance". Moreover, negative schemas or thoughts can induce depression ${ }^{5}$ or suicidal behavior ${ }^{6}$ in young persons, and negative evaluations about the self and others or negative self-concepts may be associated with the formation of delusions and hallucinations. ${ }^{7,8}$ Negative schemas are also associated with suicidal ideation in patients with psychosis ${ }^{9}$ and play a vital mediating role in the link between cyclothymic-hypersensitive temperament and 
suicidality in patients with first-episode psychosis. ${ }^{10}$

The use of neuroimaging studies to identify the neural correlates of negative schemas in patients with schizophrenia is critically important for furthering the current understanding of its pathophysiology and informing the development of future therapeutic agents. Comparisons of functional magnetic resonance imaging (fMRI) scans during self-referential versus non-self-referential tasks in healthy volunteers revealed that several cortical midline structures, including the medial prefrontal cortex (mPFC), anterior cingulate cortex (ACC), and posteromedial cortices, are key regions involved in the processing of self-referential stimuli. ${ }^{11}$ A meta-analysis of neuroimaging studies investigating neural correlates of self-reflection in schizophrenia reported that the ventral $\mathrm{mPFC}$ is responsible for tagging information relevant for 'self', whereas the dorsal mPFC is responsible for evaluation and decisionmaking processes in self- and other-referential processing. ${ }^{12}$ Other recent fMRI studies employed self-referential stimuli also demonstrated deficits in the activation of the mPFC and ACC in patients with schizophrenia. ${ }^{13,14}$ However, these studies did not address the neural correlates underlying more specific aspects of self, such as a positive or negative sense of self.

On the other hand, two previous studies may provide hints about the brain regions implicated in the perception of positive- or negative-self in patients with schizophrenia. ${ }^{15}$ presented trait adjectives that were categorized as positive, negative, mental illness-related, or physical illness-related and asked participants to judge whether these words applied to themselves, another person, or whether they contained the letter "a”. They observed hypoactivation of left superior frontal gyrus during negative self traits evaluation in patients vs. controls. ${ }^{16}$ asked people with schizophrenia and healthy controls to respond to word stimuli with a prespecified emotional valence (positive, negative, or neutral) while inhibiting responses to a stimulus with a different valence. Patients with schizophrenia were found to have failed activation of prefrontalparietal network during response inhibition to negative words. In terms of methodological aspects, the first one used adjectives, not sentences and its sample size was very small. The second one measured neural activity during response inhibition, not the activity induced by stimuli. Therefore, these two study designs appear to be insufficient to address the question at hand, and thus, further exploration of the neural substrates related to negative or positive schemas of the self and others are required.

We hypothesized that neural activation to the stimuli of negative and positive schema would be different in early stage patients with schizophrenia spectrum disorders (SSDs) compared to healthy controls. The present study aimed to identify the neural correlates associated with negative and positive schemas of the self and others between the two groups. Considering reported associations between negative evaluation and positive symptoms, ${ }^{7,8}$ the relationship between task-related activation and psychopathology was also explored. We had a special focus on studying early stage patients because of less concern with cognitive impairment, accumulative effects of antipsychotics and disease process.

\section{METHODS}

\section{Participants}

For this study, 56 individuals with patients with early stage SSD (32 with schizophrenia and 24 with schizophreniform disorder) were recruited from Jeonbuk National University Hospital, Jeonju, Republic of Korea. Early stage was defined as having a duration of illness (DI) of $\leq 5$ years. Of these 56 individuals, all were stable outpatients with no change in medication during last 3 months, and all diagnoses were made using the Structured Clinical Interview for Diagnostic and Statistical Manual of Mental Disorders, Fourth Edition Axis I Disorders (SCID-I). ${ }^{17}$ Patients with alcohol or drug use disorders within the past 6 months, an intellectual disability (IQ $\leq 70$ ), current or historical neurological disorders, a serious medical illness, pregnancy, and/or claustrophobia were excluded from the study.

Additionally, healthy participants were recruited for the control group via advertisements. These participants were age- and sex-matched to the patient group, interviewed using the axis I diagnoses with the SCID-I Non-Patient Edition (SCID-NP), ${ }^{18}$ and required to have no previous or current psychiatric disorders, neurological disorders, or significant medical conditions and no family history of psychotic disorders. All healthy controls were between 19 and 50 years of age and were assessed as right-handed using the Edinburgh Handedness Inventory. ${ }^{19}$ All participants joined this study voluntarily and provided written informed consent. The study was approved by the Ethics Committee of Jeonbuk National University Hospital (approval number: CUH 2012-08-001).

\section{Clinical assessments}

The severity of symptoms within the week prior to the fMRI experiment was evaluated in each SSD patient using the Positive and Negative Syndrome Scale, ${ }^{20}$ which was administered by trained psychiatrists. Additionally, beliefs about the self and others were evaluated with the Brief Core Schema Scales (BCSS), ${ }^{2}$ which yield subscale scores for negative-self, positive-self, negative-others, and positive-others schemas.

\section{Schema task}

The first 50 candidate sentences in the schema task described 
four schemas (negative-self, negative-others, positive-self, and positive-others) that were developed based on a classic list of personality trait words, ${ }^{21}$ the Automatic Thought Questionnaire, ${ }^{22}$ and the Affective Norms for English Words wordlist. ${ }^{23}$ The healthy controls evaluated the sentences in terms of appropriateness for schema evocation, ease of understanding, and valence using a visual analogue scale (VAS) ranging from 0 to 10 . Sentences that were rated $\geq 7$ for all items were selected, and the lengths were adjusted to be balanced over the four schemas. Finally, a psychiatrist and clinical psychologist selected 30 sentences for each schema to be used in the task.

For the neutral stimuli, 50 candidate sentences that described simple facts or knowledge were prepared. The healthy controls evaluated the sentences in terms of ease of understanding and valence. Sentences rated $\geq 7$ for ease of understanding with the VAS and those that were rated close to " 0 " for valence using an 11-point Likert scale $(-5$ to +5$)$ were selected and then finalized by the psychiatrist and clinical psychologist. Any sentences that were not selected were used for preliminary training prior to fMRI.

The fMRI procedure comprised a block design with five task conditions and one rest condition (Figure 1). An example of each condition was "I am ugly," "I am honest," "Other people are cruel," "Other people are kind," and "Rabbits are faster than turtles" for negative-self, positive-self, negative-others, positive-others and neutral condition respectively. There were five runs, with each run containing five blocks for each condition, and each block containing six trials in which a sentence was displayed for $3 \mathrm{~s}$. Participants were instructed to press the button anytime during three seconds of stimulus display. If they agreed with contents of negative- and positive-sentences or thought that contents of neutral sentences were correct, right button was pressed (vice versa). To control for order ef- fects, the blocks in each run were presented in random order with no consecutive blocks from the same condition. During the rest condition that was inserted between the task conditions, a fixation cross was shown for $18 \mathrm{~s}$. Prior to the functional run, the participants completed practice trials to become familiarized with the tasks. The total scan time for one session ( 25 blocks for the task condition and 25 rest conditions) was $15 \mathrm{~min}$.

\section{Image acquisition and preprocessing}

All fMRI data were acquired at Jeonbuk National University Hospital using a 3T Siemens Verio scanner (Siemens Medical Solutions, Erlangen, Germany) with $45 \mathrm{mT} / \mathrm{m}$ gradients and a 12-channel standard quadrature head coil. Transaxial functional scans were acquired using a single-shot $\mathrm{T} 2$ *weighted gradient-echo-planar imaging sequence that depicted the blood oxygenation level-dependent (BOLD) signal with the following parameters: repeat time $(\mathrm{TR})=3 \mathrm{~s}$, echo time $(\mathrm{TE})=30 \mathrm{~ms}$, field of view $(\mathrm{FOV})=240 \mathrm{~mm}$, acquisition matrix $=64 \times 64$, flip angle $=90^{\circ}$, gap $=0.8 \mathrm{~mm}, 302$ volumes $(36$ contiguous axial 3-mm-thick slices), and even-first interleaved acquisition. Prior to BOLD imaging, axial 1.0-mm-thick T1weighted spin-echo images (TR=1,900 ms, $\mathrm{TE}=2.5 \mathrm{~ms}$, flip angle $=9^{\circ}, \mathrm{FOV}=240 \mathrm{~mm}$, and image matrix $=256 \times 246 \mathrm{~mm}$ ) were acquired as an anatomical reference. The first three volumes were discarded to account for T1 saturation.

All processing of the fMRI data was conducted using SPM12 software (https://www.fil.ion.ucl.ac.uk/spm/software/spm12). Functional images were corrected for slice acquisition time within each volume, motion-corrected by realigning to the first volume, and co-registered with the anatomical data of each participant. Then, the functional data were transformed into a standard anatomical space based on the parameters

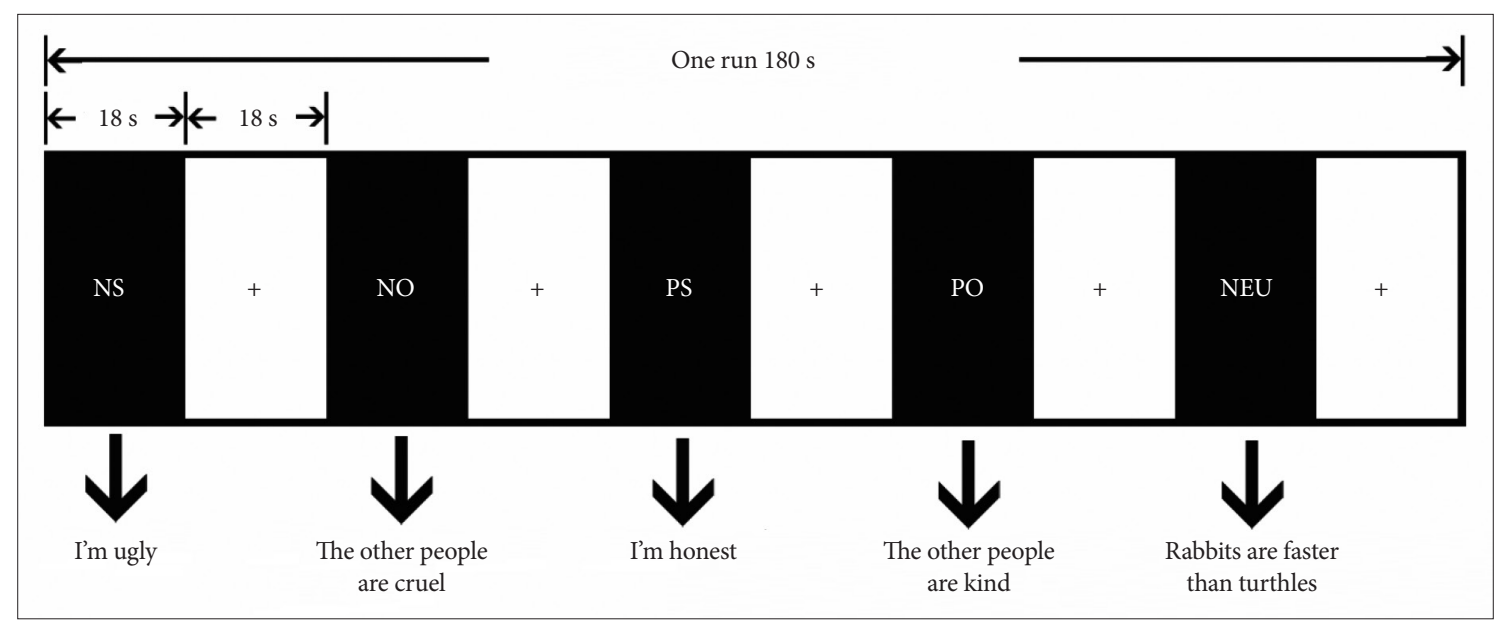

Figure 1. Task design. Every block contains six sentences each of which was presented for $3 \mathrm{~s}$. Fixation cross was shown $18 \mathrm{~s}$ between every block. The session contains five runs. The session contains five runs. NS: negative-self block, NO: negative-others block, PS: positive-self block, PO: positive-others block, NEU: neutral block, Fixation cross: rest block. 
obtained by spatially normalizing each T1 image to the Montreal Neurological Institute (MNI) template. The normalized images were smoothed with an 8-mm full-width-at-half-maximum Gaussian filter, and then a high-pass filter with a cut-off period of $128 \mathrm{~s}$ was implemented to remove low-frequency drifts from the time series. The processed data were examined for excessive motion artifacts using the Artifact Detection Tool software package (http://web.mit.edu/swg/art/art. pdf; http://www.nitrc.org/projects/artifact_detect). In total, six patients were excluded due to excessive head movements ${ }^{24}$ based on the following criteria: a maximum absolute head motion displacement of $>2 \mathrm{~mm}$ along the $\mathrm{x} / \mathrm{y} / \mathrm{z}$ axes and yaw/ pitch/role rotation $>2^{\circ}$.

\section{Analyses of the imaging and clinical data}

First-level analyses were performed for each participant and each contrast (negative-self minus neutral, negative-others minus neutral, positive-self minus neutral, and positive-others minus neutral). Then, in the second-step analyses, one-sample t-tests were performed to generate within-group activation maps, and between-group two-sample t-tests were performed for each corresponding contrast; all group comparisons included education as a covariate. As we did not have prior hypothesis, whole brain analysis was performed. Activations were considered to be significant at $\mathrm{p}$-values with a familywise error $<0.05$ (pFWE) and a cluster size threshold of $>20$ voxels. Between-group analysis was performed again with drug-naïve or drug-free patients $(n=23)$ and age and sexmatched controls $(n=23)$. Finally, in the patient group, multiple regression analyses were conducted to assess the relationships between the PANSS or BCSS score and mean activation values of the areas showing a difference under each contrasted condition using age, sex, and duration of illness as covariates. Results were considered to be significant at pFWE $<0.05$ and cluster size threshold $>20$ voxels.

To plot the correlation graphs, $\beta$-values (mean values of the areas) were extracted using MarsBaR (http://marsbar.sourceforge.net/) for significantly correlated regions. For the behavioral data, either a two-way analysis of variance (ANOVA) or t-tests were performed. When comparing two groups, either independent t-tests or chi-square tests were conducted depending on the variable type.

\section{RESULTS}

\section{Participant characteristics}

Comparison of the demographic information of SSD patients and healthy controls revealed that there was a significant difference in education level. All subscale scores on the BCSS, except for that of positive-others, differed significantly between the patients and controls (Table 1). Of the 50 SSD patients, 14 were antipsychotic-naïve, 9 were antipsychotic-

Table 1. Demographic and clinical characteristics of patients with SSDs and healthy controls

\begin{tabular}{|c|c|c|c|}
\hline Characteristics & Patient $(\mathrm{N}=50)$ & Control $(\mathrm{N}=52)$ & $\mathrm{p}$-value \\
\hline Age (years) & $29.95(7.58)$ & $31.10(5.71)$ & 0.106 \\
\hline \multicolumn{4}{|l|}{ Gender } \\
\hline Male (N) & 25 & 25 & 0.846 \\
\hline Female $(\mathrm{N})$ & 25 & 27 & \\
\hline Education (years) & $13.80(2.00)$ & $16.02(1.92)$ & $<0.001$ \\
\hline \multicolumn{4}{|l|}{ BCSS } \\
\hline Negative self & $8.32(6.62)$ & $4.27(9.55)$ & $<0.001$ \\
\hline Negative others & $7.14(6.96)$ & $1.45(2.77)$ & $<0.001$ \\
\hline Positive self & $8.84(5.64)$ & $13.29(4.93)$ & $<0.001$ \\
\hline Positive others & $7.91(6.76)$ & $9.69(5.73)$ & 0.215 \\
\hline Duration of illness (months) & $15.12(16.38)$ & - & - \\
\hline \multicolumn{4}{|l|}{ PANSS } \\
\hline Positive symptoms & $16.98(7.86)$ & - & - \\
\hline Negative symptoms & $13.84(5.50)$ & - & - \\
\hline General psychopathology & $29.16(9.82)$ & - & - \\
\hline Total symptoms & $59.98(19.45)$ & - & - \\
\hline Medication $(\mathrm{N}=27)$ & & - & - \\
\hline Chlorpromazine equivalent (mg) & $250.74(133.77)$ & - & - \\
\hline
\end{tabular}

Data given as mean (SD). SSD: schizophrenia spectrum disorders, BCSS: Brief Core Schema Scale, PANSS: Positive and Negative Syndrome Scale, SD: standard deviation 
free, and 27 were medicated. The mean chlorpromazine-equivalent dose for the patients was $250.74 \pm 133.77 \mathrm{mg} /$ day. ${ }^{25-27}$

\section{Behavioral data}

A two-way ANOVA of the reaction time (RT) data that in-

Table 2. Behavioral results of patients with SSDs and healthy controls

\begin{tabular}{lcc}
\hline & Patient & Control \\
\hline Reaction time (ms) & & \\
NS & $1530.37(262.43)$ & $1268.06(193.20)$ \\
NO & $1463.02(318.60)$ & $1335.37(216.89)$ \\
PS & $1504.52(293.91)$ & $1300.37(212.02)$ \\
PO & $1472.58(313.16)$ & $1304.12(218.28)$ \\
NEU & $1615.45(343.48)$ & $1715.59(146.40)$ \\
Accuracy for NEU (\%) & $49.81(25.49)$ & $81.89(7.51)$ \\
Overall response rate (\%) & $80.13(16.76)$ & $96.22(3.92)$ \\
\hline
\end{tabular}

Data given as mean (SD). Overall response rate: the response rate of all sentences are displayed in 15 minutes scan. SSD: schizophrenia spectrum disorders, NS: negative-self condition, NO: negativeothers condition, PS: positive-self condition, PO: positive-others condition, NEU: neutral condition, SD: standard deviation cluded group and condition type as factors revealed that the main effects of group $\left(\mathrm{F}_{1,55}=20.48, \mathrm{p}<0.001\right)$ and condition $\left(\mathrm{F}_{4,55}=13.44, \mathrm{p}<0.001\right)$ were significant, as well as the group $\times$ condition interaction $\left(\mathrm{F}_{4,55}=4.51, \mathrm{p}=0.001\right)$. Post-hoc t-tests revealed that the SSD patients exhibited significantly longer RTs under negative-self $(t=4.63, p<0.001)$, positive-self $(t=3.25$, $\mathrm{p}=0.002)$, and positive-others $(\mathrm{t}=2.54, \mathrm{p}=0.014)$ conditions compared to the healthy control group. The patients also exhibited significantly lower levels of accuracy under the neutral condition $(\mathrm{p}<0.001)$ and lower overall response rates $(\mathrm{p}<0.001)$ compared to the healthy controls (Table 2).

\section{fMRI data}

One-sample analysis

Under all contrasted conditions, activation differences were most commonly observed in the frontal gyrus and precuneus and less frequently in the temporal gyrus, cingulate gyrus, insula, or thalamus in both the patient and control groups (Figure 2); a more detailed description is provided in the Supplementary Material, Supplementary Table 1 and 2 (in the online-only Data Supplement).

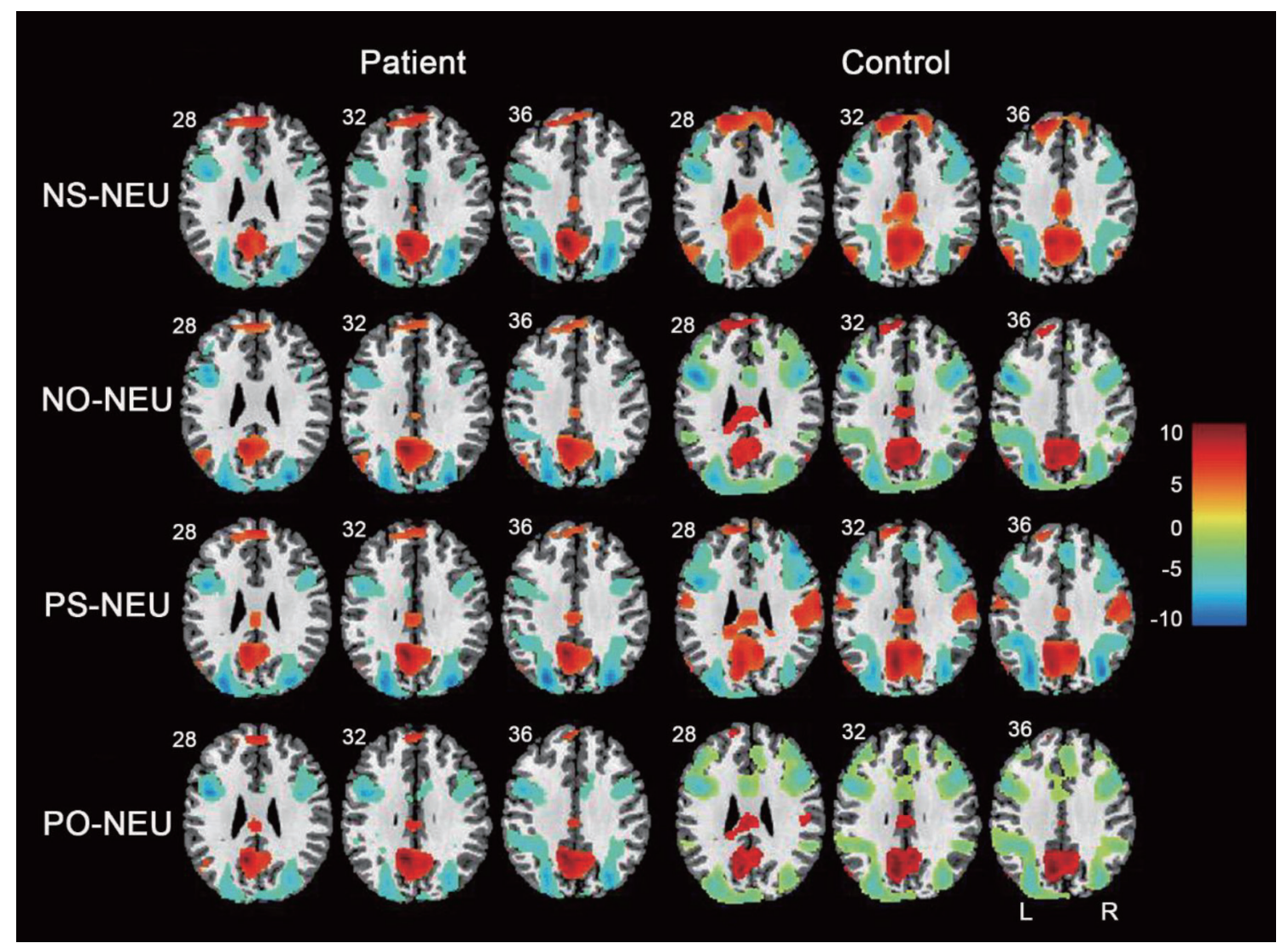

Figure 2. Brain activation during the NS-NEU, NO-NEU, PS-NEU and PO-NEU contrasts in patients and healthy controls. Left is patient group, right is healthy control group. Warm colors indicate increased activation, cool colors represent decreased activation. The number below each slice indicates slice location $(\mathrm{mm})$ of $\mathrm{MNI} z$ coordinate. NS-NEU: negative-self minus Neutral contrast, NO-NEU: negative-others minus neutral contrast, PS-NEU: positive-self minus peutral contrast, PO-NEU: positive-others minus peutral, MNI: Montreal Neurological Institute. 


\section{Group comparisons}

Negative-self minus neutral. There was no significant difference between the two groups. However, in subgroup analysis, patients with drug-naïve or drug-free state showed hyperactivation in the left superior frontal and inferior frontal gyri and hypoactivation in the right insula, and right postcentral and parahippocampal gyri (Supplementary Table 3 in the online-only Data Supplement).

\section{Negative-others minus neutral}

The SSD patients exhibited relatively higher activity in the left precentral gyrus ( $t=4.73$, MNI coordinates: $-46,8,36)$, left inferior frontal gyrus $(\mathrm{t}=3.74$, MNI coordinates: $-50,14,28)$, and left middle frontal gyrus ( $\mathrm{t}=3.61$, MNI coordinates: -30 , $14,36)$ compared to the healthy controls. In subgroup analysis, patients showed hyperactivation in the right anterior cingulate gyrus and cerebellum crus 2 (Supplementary Table 3 in the online-only Data Supplement).

\section{Positive-self minus neutral}

The SSD patients exhibited significantly higher activity in the left superior frontal gyrus ( $\mathrm{t}=3.59$, MNI coordinates: -14 , $44,24)$, left middle frontal gyrus ( $\mathrm{t}=3.13$, MNI coordinates: $-20,42,24)$, and right medial superior frontal gyrus ( $t=2.72$, MNI coordinates: 8, 48, 32) compared to the healthy controls. Additionally, the SSD patients exhibited significantly lower activity in the right postcentral gyrus ( $\mathrm{t}=4.66, \mathrm{MNI}$ coordinates: $36,-30,56)$, right precentral gyrus $(\mathrm{t}=4.23$, MNI coordinates: $42,-14,58)$, right Rolandic operculum gyrus ( $\mathrm{t}=3.52$, MNI coordinates: $52,-28,20)$, and right supplementary motor area $(\mathrm{t}=2.82$, MNI coordinates: $14,-18,64)$ compared to the healthy controls. In subgroup analysis, patients showed hyperactivation in the right superior frontal, inferior frontal operculum, middle temporal gyri and left precuneus (Supplementary Table 3 in the online-only Data Supplement).

\section{Positive-others minus neutral}

The SSD patients exhibited significantly (pFWE $<0.05$, cluster size $>20$ ) higher activity in the left superior frontal gyrus $(t=3.42$, MNI coordinates: $-14,44,24)$, left middle frontal gyrus ( $\mathrm{t}=3.40$, MNI coordinates: $-20,28,34)$, and right medial superior frontal gyrus ( $\mathrm{t}=3.36$, MNI coordinates: $10,36,44)$ compared to the healthy controls (Table 3, Figure 3). In subgroup analysis, patients showed hyperactivation in the cerebellum 6 and crus 1 (Supplementary Table 3 in the online-only Data Supplement).

\section{Correlations}

Under the positive-others minus neutral contrast, there were significant negative correlations $(\mathrm{r}=-0.567, \mathrm{p}<0.05)$ between the PANSS total score and activation at the regional cluster including right angular gyrus (AG; MNI coordinates: 38, -64,

Table 3. Results of group comparisons in different contrasts

\begin{tabular}{|c|c|c|c|c|c|}
\hline Contrast & Brain region & Cluster size & $\begin{array}{c}\text { MNI coordinates } \\
\text { of peak voxel }\end{array}$ & $\mathrm{t}$-value & pFWE corrected \\
\hline \multirow[t]{4}{*}{ Negative-others minus neutral } & Patient $>$ control & & & & \\
\hline & Left precentral gyrus & 986 & $-46,8,36$ & 4.73 & 0.013 \\
\hline & Left inferior frontal gyrus & & $-50,14,28$ & 3.74 & \\
\hline & Left middle frontal gyrus & & $-30,14,36$ & 3.61 & \\
\hline \multirow[t]{9}{*}{ Positive-self minus neutral } & Patient $>$ control & & & & \\
\hline & Left superior frontal gyrus & 1,116 & $-14,44,24$ & 3.59 & 0.007 \\
\hline & Left middle frontal gyrus & & $-20,42,24$ & 3.13 & \\
\hline & Right medial superior frontal gyrus & & $8,48,32$ & 2.72 & \\
\hline & Patient $<$ control & & & & \\
\hline & Right postcentral gyrus & 4,196 & $36,-30,56$ & 4.66 & $<0.001$ \\
\hline & Right precentral gyrus & & $42,-14,58$ & 4.23 & \\
\hline & Right rolandic operculum gyrus & & $52,-28,20$ & 3.52 & \\
\hline & Right supplementary motor area & & $14,-18,64$ & 2.82 & \\
\hline \multirow[t]{4}{*}{ Positive-others minus neutral } & Patient $>$ control & & & & \\
\hline & Left superior frontal gyrus & 648 & $-14,44,24$ & 3.42 & 0.044 \\
\hline & Left middle frontal gyrus & & $-20,28,34$ & 3.40 & \\
\hline & Right medial superior frontal gyrus & & $10,36,44$ & 3.36 & \\
\hline
\end{tabular}

Whole-brain thresholded at $\mathrm{p}<0.05$, FEW corrected with a voxel extent of $>20$; Only coordinates of the highest peak in each cluster are reported. MNI: Montreal Neurological Institute, pFWE: probability family wise error 


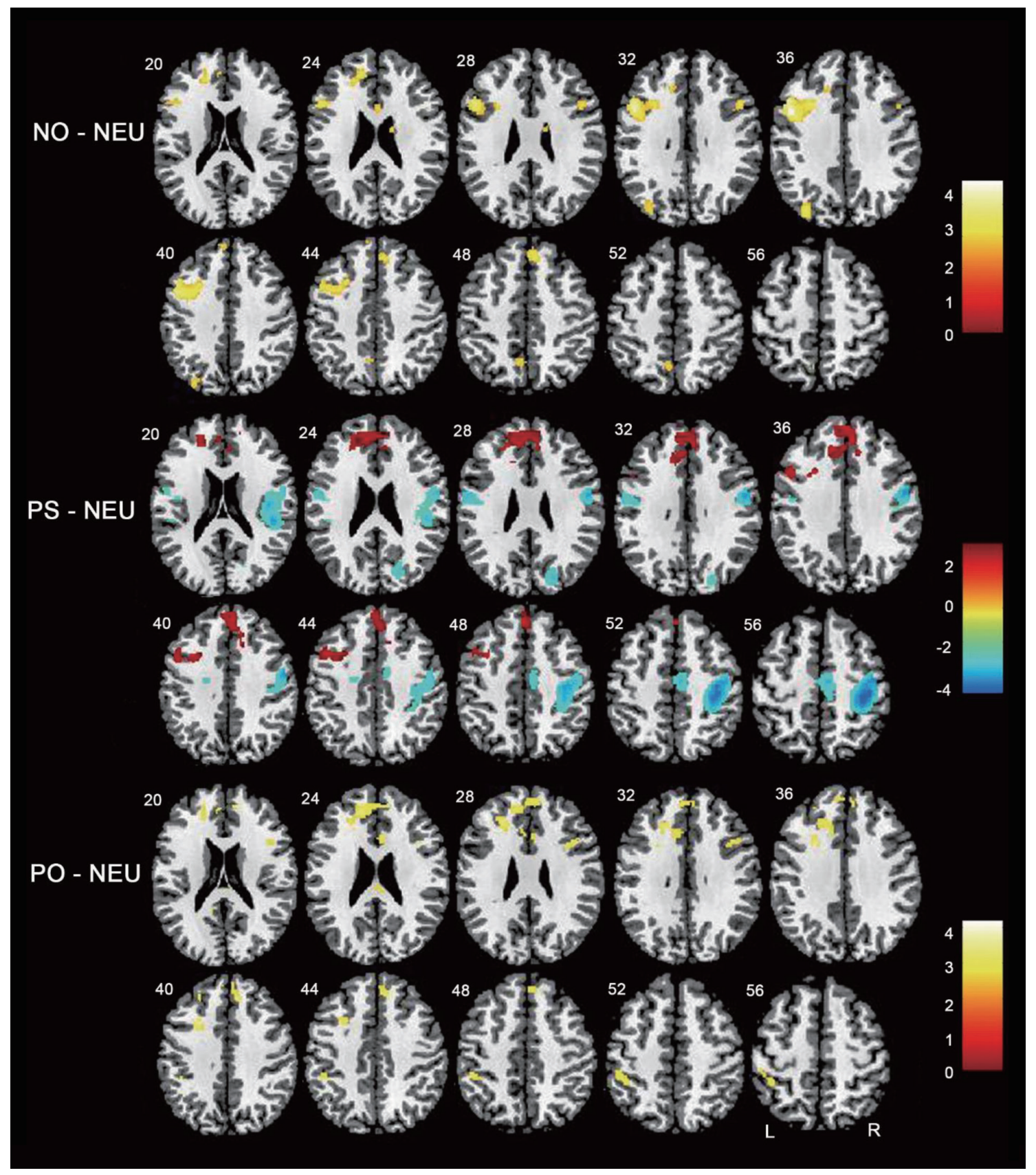

Figure 3. Comparison between patients and healthy controls in the NO-NEU, PS-NEU and PO-NEU contrasts. Warm colors indicate greater activation in patients than controls whereas cool colors represent decreased activation in patients. The number below each slice indicates slice location $(\mathrm{mm})$ of MNI z coordinate. NO-NEU: negative-others minus neutral contrast, PS-NEU: positive-self minus neutral contrast, PO-NEU: positive-others minus neutral contrast, MNI: Montreal Neurological Institute.

50) and right inferior parietal gyrus (MNI coordinates: 40, -54 , 52). For the BCSS, the positive-others score was significantly and positively correlated $(\mathrm{r}=0.497, \mathrm{p}<0.05)$ with activation at the regional cluster including left middle cingulate gyrus (MNI coordinates: $-8,-44,54$ ), right cuneus (MNI coordinates: 16 , $-70,36$ ), left precuneus (MNI coordinates: 10, $-50,52$ ) and right precuneus (MNI coordinates: 6, $-42,54$ ) under the same contrast (Figure 4).

\section{DISCUSSION}

Patients with schizophrenia often have deep-rooted negative-self or -others schemas that are closely associated with the formation of positive symptoms and suicidality during psychosis. Thus, the present study was based on the assumption that there would be differences in brain activation in response to self- or others-related negative and positive sentences be- 

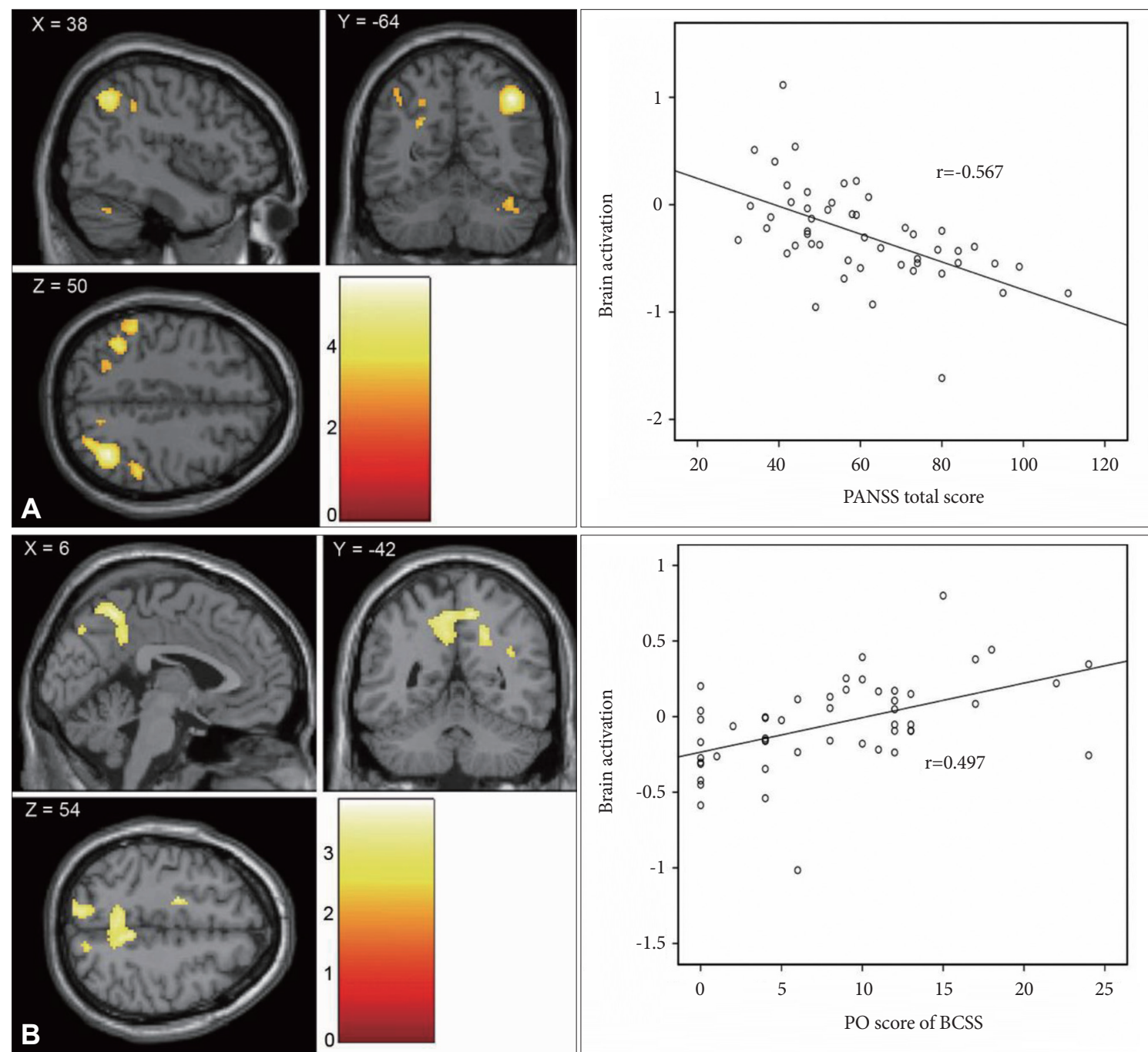

Figure 4. Correlation between the PANSS total score or BCSS score and brain activation during the positiveothers minus neutral contrast in patients with SSD. A: Negative correlation of the PANSS total score with the activity in the right angular gyrus and right inferior parietal gyrus. B: Positive correlation of positive-others score of the BCSS with the activity in the left middle cingulate gyrus, right cuneus and left/ right precuneus. PANSS: Positive and Negative Syndrome Scale, BCSS: Brief Core Schema Scale, SSD: schizophrenia spectrum disorders.

tween SSD patients and healthy controls.

In the present study, the SSD patients showed relatively higher activity in the left middle and inferior frontal gyri and left precentral gyrus compared to the healthy controls under the negative-others minus neutral contrast. It should be noted that percent signal changes of contrast images in both patients and controls were minus values and its value was greater in controls (data not shown). Therefore, it seems that higher activity in patients can be interpreted as less deactivation under this contrast compared to controls. As both patients and controls showed negative percent signal changes under negative-others condition, this suggests that task of negative-others condition may be associated with neuronal inhibition which may in turn decrease BOLD signal. This speculation may be supported by the finding that negative contrast values were observed in the right middle and superior frontal gyri of pa- tients with schizophrenia during response inhibition to negative compared with neutral words. ${ }^{16}$ However, in that same study ${ }^{16}$ healthy controls showed positive contrast values which is opposite to our result. It has been reported that in healthy controls, negative self-related sentences induce hyperactivation of the dorsomedial prefrontal cortex (dmPFC), ACC, and insula ${ }^{28}$ and forgetting negative self-referential information is associated with more widespread activation that includes the middle frontal gyrus and inferior frontal gyrus. ${ }^{29}$ Direct comparison of these results should be cautious given that details of the tasks and participant's characteristics were different. In order to deepen understanding and implication of related findings, percent signal change of each condition needs to be verified which most of studies seldom provide. Nevertheless, relatively higher activity in the middle/inferior frontal gyrus and precentral gyrus of schizophrenia patients during nega- 
tive-other minus neutral contrast resonates with the findings of two previous studies: relatively higher activity in the right superior and middle frontal gyri of patients under the refusal minus acceptance condition ${ }^{30}$ and relative overactivity in the left superior frontal gyrus of patients during self-evaluation in response to negative trait adjectives. ${ }^{15}$ Interpretation of less deactivation to negative stimuli in patients compared to controls may be that neural plasticity in patients is so rigid that inefficient response to negative stimuli may occur in patients. It should be noted that we did not observe significant difference of brain activation between the two groups under the negative-self minus neutral contrast despite of significantly higher score of negative-self in patients compared to controls. One explanation for this discrepant finding may be that mental states of patients could have been different between the time points of scan and assessment of the BCSS performed. Alternatively, actual environmental conditions for scanning and scale assessment are quite different that the results from the two conditions do not necessarily have to be the same. Last but interesting possibility could be related to the results of subgroup analysis. As drug-naïve/free patients showed significantly different activations under negative-self minus neutral contrast, antipsychotics may have exerted dampening effect on brain activation but not on the scale performance.

In the present study, the SSD patients exhibited greater activation in the left superior and middle frontal gyri and right medial superior frontal gyrus than the healthy controls in the positive-self and -others minus neutral contrasts. These findings are in contrast with those of Bedford et al., ${ }^{15}$ who observed no significant findings in patients with schizophrenia compared to controls during self-evaluation in response to positive trait adjectives. Other studies have reported that control participants exhibit increased activity in the right dmPFC and left ventromedial PFC in response to positive self-related sentences or words. ${ }^{28,31}$ Therefore, the present results appear to be counterintuitive. It is possible that repulsion towards positive descriptions of the self or others may have played a role in the induction of greater activation of the frontal regions of SSD patients compared to controls. Alternatively, it may suggest that patients need more blood perfusion to process positive stimuli because of inefficient neural mechanisms. Previous findings showing that people with schizophrenia do not deactivate the cingulate areas during response inhibition to positive words ${ }^{16}$ partially support this notion. Moreover, the precentral gyrus and postcentral gyrus are primary sensory and motor cortices, respectively, ${ }^{32}$ and are typically active during any task that involves movement. ${ }^{33}$ Likewise, the Rolandic operculum gyrus is activated during visual stimulation. ${ }^{34}$ The present findings showing lower levels of activity in the pre- and postcentral gyri, the Rolandic operculum gyrus, and supplementary motor areas in SSD patients may have been due to their slow sensorimotor responses, which was reflected in the longer RTs for pressing the button.

In the present study, correlation analyses revealed negative relationships between the PANSS total score and activity in the right angular and inferior parietal gyri. The AG is involved in semantic processing, word reading and comprehension, number processing, the default mode network, memory retrieval, attention, spatial cognition, reasoning, and social cognition. ${ }^{35}$ The role of the inferior parietal lobe (IPL) is to maintain attentive control during current task goals as well as to respond to salient novel information or alerting stimuli in the environment. ${ }^{36}$ The percent signal changes in the angular and parietal gyri under the positive-others condition were negative values suggesting presence of deactivation. Based on the functional roles of the AG and IPL and their negative signal changes, it is possible that more severe abnormalities in these regions may result in a greater degree of dysregulation in terms of neurocognition and social cognition, which, in turn, could manifest as a more severe psychopathology.

Interestingly, in the present study, the positive-others score on the BCSS was positively correlated with activity in the left middle cingulate gyrus and left or right precuneus/cuneus. The cingulate cortex processes cognitive, emotional, and social information. ${ }^{37}$ The middle cingulate cortex (MCC) is the dorsal portion of the ACC and has the highest level of connectivity with areas related to cognitive (dorsal PFC) and motor (premotor and motor cortices) functions. ${ }^{38}$ The precuneus is involved in a wide variety of highly integrated tasks such as visuospatial imagery, episodic memory retrieval, and self-processing operations, including taking a first-person perspective and the experience of agency. ${ }^{39}$ The cuneus (Brodmann area 17) receives visual information from the same-sided superior quadrantic retina and is primarily involved in basic visual processing. Taken together, these findings indicate that higher activities in the MCC and precuneus are associated with a greater degree of taking a positive perspective towards others, even though the positive-others score was low for the SSD patients in this study. Its implication may be that any interventions, drugs or transmagnetic brain stimulation, targeting to enhance blood perfusion or neural activity in the MCC and precuneus would be helpful in treating patients. On the other hand, it was unexpected results not observing significant correlation with other contrasts given that the scores of negativeself and negative-others in patients were significantly higher than controls. Considering that total score of the PANSS in patients was relatively low, it may be that performing the task of positive-others rather than negative related task might have been more strenuous which may be related to the severity of psychopathology. 
The present study has several limitations that should be considered. First, the participants were stable and exhibited mild levels of symptomatology and thus, the PANSS may not be sufficiently sensitive to detect correlations between its subscale scores and activation signals. The use of more detailed and comprehensive scales, such as the Psychotic Symptom Rating Scales, ${ }^{40}$ may produce different results. Second, although education was included as a covariate in the analyses, this does not rule out the possible contribution of education to the group differences in regional activation. Thus, a more careful recruitment process for healthy control participants will be necessary in the future. Third, most patients in the present study were medicated and this fact should be considered when interpreting the results. Despite these caveats, the present study is the first to provide evidence of the neural signatures related to negative and positive schemas towards the self and others in SSD patients.

In conclusion, the present negative and positive schema task revealed that SSD patients exhibited significantly different activations under the negative-others minus neutral contrast and positive-self and -others minus neutral contrasts compared to healthy controls. These findings suggest that the frontal regions are more sensitive and showing inefficient response to negative and positive schemas of the self and/or others in SSD patients compared to healthy subjects.

\section{Supplementary Materials}

The online-only Data Supplement is available with this article at https://doi.org/10.30773/pi.2020.0335.

\section{Acknowledgments}

The corresponding author would like to thank all participants in the study and the Father in Heaven. The authors report no biomedical financial interests or potential conflicts of interest.

This study was supported by a grant from the Korean Mental Health Technology R\&D Project, Ministry of Health \& Welfare, Republic of Korea (HL19C0015) and a grant from the Korea Health Technology R\&D Project through the Korea Health Industry Development Institute, which is funded by the Ministry of Health \& Welfare, Republic of Korea (HI18C2383).

\section{Conflicts of Interest}

The authors have no potential conflicts of interest to disclose.

\section{Author Contributions}

Conceptualization: Young-Chul Chung. Data curation: Young-Chul Chung, Jie Shen. Formal analysis: Jie Shen, Guangfan Shen. Funding acquisition: Young-Chul Chung. Investigation: Guangfan Shen. Methodology: Woo-Sung Kim, Jie Shen. Project administration: Woo-Sung Kim, Congcong Liu, Guangfan Shen, Uyanga Tsogt. Resources: Woo-Sung Kim. Software: Woo-Sung Kim. Supervision: Young-Chul Chung. Validation: Young-Chul Chung. Visualization: Guangfan Shen. Writing-original draft: Guangfan Shen, Jie Shen. Writing - review \& editing: Young-Chul Chung.

\section{ORCID iDs}

\section{Jie Shen}

Guangfan Shen

https://orcid.org/0000-0002-5479-9175

https://orcid.org/0000-0003-4555-1781
Woo-Sung Kim

Uyanga Tsogt

Congcong Liu

Jing Sui

Young-Chul Chung

\section{REFERENCES}

1. Henriksen MG, Parnas J. Self-disorders and schizophrenia: a phenomenological reappraisal of poor insight and noncompliance. Schizophr Bull 2014;40:542-547.

2. Fowler D, Freeman D, Smith B, Kuipers E, Bebbington P, Bashforth H, et al. The Brief Core Schema Scales (BCSS): psychometric properties and associations with paranoia and grandiosity in non-clinical and psychosis samples. Psychol Med 2006;36:749-759.

3. Young JE. Cognitive Therapy for Personality Disorders: A Schema-Focused Approach (Rev. ed.). Sarasota, FL: Professional Resource Press; 1999.

4. Lumley MN, Harkness KL. Specificity in the relations among childhood adversity, early maladaptive schemas, and symptom profiles in adolescent depression. Cogn Ther Res 2007;31:639-657.

5. Balsamo M, Carlucci L, Sergi MR, Klein, Murdock K, Saggino A. The mediating role of early maladaptive schemas in the relation between corumination and depression in young adults. PLoS One 2015;10:e0140177.

6. Choon MW, Talib MA, Yaacob SN, Awang H, Tan JP, Hassan S, et al. Negative automatic thoughts as a mediator of the relationship between depression and suicidal behaviour in an at-risk sample of Malaysian adolescents. Child Adolesc Ment Health 2015;20:89-93.

7. Garety PA, Kuipers E, Fowler D, Freeman D, Bebbington PE. A cognitive model of the positive symptoms of psychosis. Psychol Med 2001; 31:189-195.

8. Smith B, Fowler DG, Freeman D, Bebbington P, Bashforth H, Garety P, et al. Emotion and psychosis: Links between depression, self-esteem, negative schematic beliefs and delusions and hallucinations. Schizophr Res 2006;86:181-188.

9. Fialko L, Freeman D, Bebbington PE, Kuipers E, Garety PA, Dunn G, et al. Understanding suicidal ideation in psychosis: findings from the Psychological Prevention of Relapse in Psychosis (PRP) trial. Acta Psychiatr Scand 2006; 114:177-186.

10. Cui Y, Kim SW, Lee BJ, Kim JJ, Yu JC, Lee KY, et al. Negative schema and rumination as mediators of the relationship between childhood trauma and recent suicidal ideation in patients with early psychosis. J Clin Psychiatry 2019;80:17m12088.

11. Northoff G, Bermpohl F. Cortical midline structures and the self. Trends Cogn Sci 2004;8:102-107.

12. Van der Meer L, Costafreda S, Aleman A, David AS. Self-reflection and the brain: a theoretical review and meta-analysis of neuroimaging studies with implications for schizophrenia. Neurosci Biobehav Rev 2010;34: 935-946.

13. Pauly KD, Kircher TT, Schneider F, Habel U. Me, myself and I: temporal d ysfunctions during self-evaluation in patients with schizophrenia. Soc Cogn Affect Neurosci 2014;9:1779-1788.

14. Spaniel F, Tintera J, Rydlo J, Ibrahim I, Kasparek T, Horacek J, et al. Altered neural correlate of the self-qgency experience in first-episode schizophrenia-spectrum patients: an fMRI study. Schizophr Bull 2016; 42:916-925.

15. Bedford NJ, Surguladze S, Giampietro V, Brammer MJ, David AS. Selfeval uation in schizophrenia: an fMRI study with implications for the understanding of insight. BMC Psychiatry 2012;12:106.

16. Vercammen A, Morris R, Green MJ, Lenroot R, Kulkarni J, Carr VJ, et al. Reduced neural activity of the prefrontal cognitive control circuitry during response inhibition to negative words in people with schizophrenia. J Psychiatry Neurosci 2012;37:379-388.

17. American Psychiatric Association. Diagnostic and Statistical Manual of Mental Disorders: DSM-5. Arlington, VA: American Psychiatric Asso- 
ciation; 2013.

18. Han OS, Hong JP. Structured Clinical Interview for DSM-IV Axis Disorders, Research Version. Seoul: Korea Hana Medical Publishing; 2000.

19. Oldfield RC. The assessment and analysis of handedness: the Edingburgh inventory. Neuropsychologia 1971;9:97-113.

20. Kay SR, Fiszbein A, Opler LA. The positive and negative syndrome scale (PANSS) for schizophrenia. Schizophr Bull 1987;13:261-276.

21. Anderson NH. Likableness ratings of 555 personality-trait words. J Pers Soc Psychol 1968;9:272-279.

22. Hollon SD, Kendall PC. Cognitive self-statements in depression: development of an automatic thoughts questionnaire. Cogn Ther Res 1980; 4:383-395.

23. Bradley MM, Lang PJ. Affective Norms for English Words (ANEW): Instruction Manual and Affective Ratings. Technical Report C-1, The Center for Research in Psychophysiology. Florida: University of Florida; 1999.

24. Heleven E, Overwalle FV. The person within: memory codes for persons and traits using fMRI repetition suppression. Soc Cogn Affect Neurosci 2016;11:159-171.

25. Arakawa R, Ito H, Takano A, Takahashi H, Morimoto T, Sassa T, et al. Dose-finding study of paliperidone ER based on striatal and extrastriatal dopamine D2 receptor occupancy in patients with schizophrenia. Psychopharmacology 2008;197:229-235.

26. Gardner DM, Murphy AL, O’Donnell H, Centorrino F, Baldessarini RJ. International consensus study of antipsychotic dosing. Am J Psychiatry 2010;167:686-693.

27. Hales RE, Yudofsky SC, Gabbard GO. The American Psychiatric Publishing Textbook of Psychiatry (5th ed), Washington, DC: American Psychiatric Publishing, 2008, p.1086.

28. Modinos G, Renken R, Ormel J, Aleman A. Self-reflection and the psychosis-prone brain: an fMRI study. Neuropsychology 2011;25:295-305.

29. Yang W, Liu P, Cui Q, Wei D, Li W, Qiu J, et al. Directed forgetting of negative self-referential information is difficult: an FMRI study. PLoS
One 2013;8:e75190

30. Lee HR, Ku JH, Kim JH, Jang DP, Yoon KJ, Kim SI, et al. Aberrant neural responses to social rejection in patients with schizophrenia. Soc Neuro Sci 2014;9:412-423.

31. Fossati P, Hevenor SJ, Graham SJ, Grady C, Keightley ML, Craik F, et al. In search of the emotional self: an fMRI study using positive and negative emotional words. Am J Psychiatry 2003;160:1938-1945.

32. Johns P. Clinical Neuroscience E-Book: An Illustrate Colour Text, 2014, p.32.

33. Fiez JA. Reading, Neural Basis of. In: Fiez JA, Smelser NJ, Baltes PB, Editors. International Encyclopedia of the Social \& Behavioral Sciences. Canada: Elsevier, 2001, p.12791-12797.

34. Blefari ML, Martuzzi R, Salomon R, Bello-Ruiz J, Herbelin B, Serino A, et al. Bilateral Rolandic operculum processing underlying heartbeat awareness reflects changes in bodily self-consciousness. Eur J Neurosci 2017;45:1300-1312.

35. Seghier ML. The angular gyrus: multiple functions and multiple subdivisions. Neuroscientist 2013;19:43-61.

36. Singh-Curry V, Husain M. The functional role of the inferior parietal lobe in the dorsal and ventral stream dichotomy. Neuropsychologia 2009;47:1434-1448.

37. Hadland KA, Rushworth MF, Gaffan D, Passingham RE. The effect of cingulate lesions on social behaviour and emotion. Neuropsychologia 2003;41:919-931.

38. Stevens FL, Hurley RA, Taber KH. Anterior cingulate cortex: unique role in cognition and emotion. J Neuropsychiatry Clin Neurosci 2011; 23:121-125.

39. Cavanna AE, Trimble MR. The precuneus: a review of its functional anatomy and behavioural correlates. Brain 2006;129:564-583.

40. Haddock G, McCarron J, Tarrier N, Faragher EB. Scales to measure dimensions of hallucinations and delusions: the psychotic symptom rating scales (PSYRAT). Psychol Med 1999;29:879-889. 


\title{
SUPPLEMENTARY MATERIAL
}

\section{One-sample analysis}

\author{
One-sample t-tests were performed for the within-group analyses under task minus neutral conditions
}

\section{In patients}

1) Under the negative-self minus neutral condition, the left and right precuneus, the right medial superior frontal gyrus and left superior frontal gyrus exhibited increased activation, whereas the left inferior temporal gyrus, left superior occipital gyrus, left inferior parietal gyrus, left inferior opercular frontal gyrus, left inferior triangle frontal gyrus, left middle frontal gyrus, right superior frontal gyrus, right precentral gyrus, and right inferior triangle frontal gyrus exhibited decreased activation;

2) Under the negative-others minus neutral condition, the left precuneus, left angular gyrus, left middle temporal gyrus, and left and right medial superior frontal gyrus exhibited increased activation, whereas the right calcarine, right middle occipital gyrus, left cuneus, left inferior opercular frontal gyrus, left superior frontal gyrus, left inferior triangle frontal gyrus, right precentral gyrus, right superior frontal gyrus, and right supplementary motor area exhibited decreased activation;

3) Under the positive-self minus neutral condition, the left posterior cingulate gyrus, right posterior cingulate gyrus, left medial superior frontal gyrus, right medial superior frontal gyrus, and left middle cingulate gyrus exhibited increased activation, whereas the left superior occipital gyrus, right superior occipital gyrus, left inferior temporal gyrus, left inferior triangle frontal gyrus, right superior frontal gyrus, right insula, left inferior opercular frontal gyrus, and left middle frontal gyrus exhibited decreased activation; and

4) Under the positive-others minus neutral condition, the left and precuneus, right medial superior frontal gyrus, and left medial superior frontal gyrus exhibited increased activation, whereas the left inferior temporal gyrus, right fusiform gyrus, right superior occipital gyrus, left inferior opercular frontal gyrus, left superior frontal gyrus, right precentral gyrus, right inferior triangle frontal gyrus, right precentral gyrus, left thalamus, and right middle cingulate gyrus exhibited decreased activation.

\section{In healthy controls}

1) Under the negative-self minus neutral condition, the left medial orbital frontal gyrus, left medial superior frontal gyrus, left anterior cingulate gyrus, left precuneus, right middle cingulate gyrus, and left angular gyrus exhibited increased activation, whereas the right insula, right superior frontal gyrus, right meddle frontal gyrus, right angular gyrus, left inferior parietal gyrus, right hippocampus, and right calcarine exhibited decreased activation;

2) Under the negative-others minus neutral condition, the left and right precuneus, right medial superior frontal gyrus, and left medial superior frontal gyrus exhibited increased activation, whereas the left precentral gyrus, left insula, right inferior triangle frontal gyrus, left calcarine, and right anterior cingulate gyrus exhibited decreased activation;

3) Under the positive-self minus neutral condition, the right and left precuneus and left medial superior frontal gyrus exhibited increased activation, whereas the left inferior parietal gyrus, left superior parietal gyrus, right insula, right middle frontal gyrus, right opercular inferior frontal gyrus, left superior frontal gyrus, left middle frontal gyrus, and left insula exhibited decreased activation; and

4) Under the positive-others minus neutral condition, the left and right precuneus, right insula, and right and left medial orbital frontal gyri exhibited increased activation, whereas the left inferior temporal gyrus exhibited decreased activation.

\section{One-sample t-tests were performed for the within-group analyses under task minus rest conditions}

\section{In patients}

1) Under the negative-self minus rest condition, the right meddle occipital gyrus, right superior parietal gyrus and left middle frontal gyrus exhibited decreased activation;

2) Under the negative-others minus rest condition, the left precuneus and left middle cingulate gyrus exhibited decreased activation;

3) Under the positive-self minus rest condition, the left precuneus and left middle occipital gyrus exhibited decreased activation;

4) Under the positive-others minus rest condition, the left fusiform gyrus exhibited increased activation, whereas the left middle frontal gyrus, left superior frontal gyrus, left middle cingulate gyrus and left middle occipital gyrus exhibited decreased 
activation.

5) Under the neutral minus rest condition, the left lingual gyrus and left middle occipital gyrus exhibited increased activation, whereas the left precuneus exhibited decreased activation.

\section{In healthy controls}

1) Under the negative-self minus rest condition, the left middle occipital gyrus and right lingual gyrus exhibited increased activation, whereas the left caudate, eft middle occipital gyrus, right cuneus, right opercular inferior frontal gyrus, right superior frontal gyrus, left middle frontal gyrus and left triangle inferior frontal gyrus exhibited decreased activation;

2) Under the negative-others minus rest condition, the left middle occipital gyrus exhibited increased activation, whereas the left caudate, right cuneus, left middle occipital gyrus, right middle occipital gyrus, left middle frontal gyrus, right and left triangle inferior frontal gyrus, right orbit medial frontal gyrus, right media superior frontal gyrus, right opercular inferior frontal gyrus and right superior frontal gyrus exhibited decreased activation;

3) Under the positive-self minus rest condition, the left and right insula, right opercular Rolandic gyrus, left middle occipital gyrus and left inferior occipital gyrus exhibited increased activation, whereas the left and right middle occipital gyrus, left caudate, left and right middle frontal gyrus, right superior frontal gyrus, left and right orbit medial frontal gyrus exhibited decreased activation; and

4) Under the positive-others minus rest condition, the left middle occipital gyrus and right lingual gyrus exhibited increased activation, whereas the left caudate, left and right middle occipital gyrus, right superior frontal gyrus, right opercular inferior frontal gyrus, and right triangle inferior frontal gyrus exhibited decreased activation.

5) Under the neutral minus rest condition, the left middle occipital gyrus exhibited increased activation, whereas the left caudate, right cuneus, left and right middle frontal gyrus, right medial superior frontal gyrus, right superior frontal gyrus exhibited decreased activation.

\section{Two-sample analysis}

Two-sample t-tests were performed for the within-in drug-naïve/drug-free patients group analyses under task minus neutral conditions.

1) Under the negative-self minus neutral condition, in subgroup analysis, patients with drug-naïve or drug-free state showed hyperactivation in the left superior frontal and inferior frontal gyri and hypoactivation in the right insula, and right postcentral and parahippocampal gyri;

2) Under the negative-others minus neutral condition, In subgroup analysis, patients showed hyperactivation in the right anterior cingulate gyrus and cerebellum crus 2;

3) Under the positive-self minus neutral condition, In subgroup analysis, patients showed hyperactivation in the right superior frontal, inferior frontal operculum, middle temporal gyri and left precuneus; and

4) Under the positive-others minus neutral condition, In subgroup analysis, patients showed hyperactivation in the cerebellum 6 and crus 1. 


\begin{tabular}{|c|c|c|c|c|c|}
\hline Task & Brain region & Cluster size & $\begin{array}{c}\text { MNI coordinates } \\
\text { of peak voxel }\end{array}$ & $\mathrm{t}$-value & pFWE corrected \\
\hline \multirow[t]{15}{*}{$\overline{\mathrm{NS}-\mathrm{NEU}}$} & Increase activation & & & & \\
\hline & Left precuneus & 1,127 & $-8,-54,34$ & 7.79 & 0.000 \\
\hline & Right precuneus & & $6,-56,32$ & 5.41 & \\
\hline & Right medial superior frontal gyrus & 954 & $4,54,26$ & 5.57 & 0.001 \\
\hline & Left superior frontal gyrus & & $-20,48,38$ & 5.32 & \\
\hline & Decrease activation & & & & \\
\hline & Left inferior temporal gyrus & 22,360 & $-52,-50,-10$ & 8.10 & 0.000 \\
\hline & Left superior occipital gyrus & & $-26,-72,38$ & 8.10 & \\
\hline & Left inferior parietal gyrus & & $-38,-44,42$ & 7.61 & \\
\hline & Left inferior opercular frontal gyrus & 3,857 & $-40,6,26$ & 6.76 & 0.000 \\
\hline & Left inferior triangle frontal gyrus & & $-44,34,22$ & 6.25 & \\
\hline & Left middle frontal gyrus & & $-28,-6,52$ & 6.03 & \\
\hline & Right superior frontal gyrus & 2,334 & $30,2,62$ & 5.84 & 0.000 \\
\hline & Right precentral gyrus & & $30,-6,52$ & 5.77 & \\
\hline & Right inferior triangle frontal gyrus & & $54,18,22$ & 5.02 & \\
\hline \multirow[t]{16}{*}{ NO-NEU } & Increase activation & & & & \\
\hline & Left precuneus & 1,447 & $-8 ;-56 ; 30$ & 8.87 & 0.000 \\
\hline & Left angular gyrus & 354 & $-48 ;-70 ; 34$ & 6.71 & 0.031 \\
\hline & Left middle temporal gyrus & & $-54,-66,22$ & 5.03 & \\
\hline & Left medial superior frontal gyrus & 1,026 & $-12,28,58$ & 6.03 & 0.000 \\
\hline & Right medial superior frontal gyrus & & $10,50,20$ & 5.61 & \\
\hline & Decrease activation & & & & \\
\hline & Right calcarine & 17,660 & $12,-78,8$ & 7.79 & 0.000 \\
\hline & Right middle occipital gyrus & & $30,-82,30$ & 7.38 & \\
\hline & Left cuneus & & $2,-94,14$ & 7.05 & \\
\hline & Left inferior opercular frontal gyrus & 2,578 & $-40,6,26$ & 6.30 & 0.000 \\
\hline & Left superior frontal gyrus & & $-26,-8,54$ & 5.95 & \\
\hline & Left inferior triangle frontal gyrus & & $-46,30,22$ & 5.61 & \\
\hline & Right precentral gyrus & 579 & $32,-6,52$ & 5.05 & 0.005 \\
\hline & Right superior frontal gyrus & & $26,2,60$ & 4.48 & \\
\hline & Right supplementary motor area & & $16,-10,64$ & 3.65 & \\
\hline \multirow[t]{15}{*}{ PS-NEU } & Increase activation & & & & \\
\hline & Left posterior cingulate gyrus & 1,254 & $-8,-54,32$ & 8.57 & 0.000 \\
\hline & Right posterior cingulate gyrus & & $8,-54,30$ & 5.55 & \\
\hline & Left medial superior frontal gyrus & 807 & $-4,54,26$ & 5.94 & 0.000 \\
\hline & Right medial superior frontal gyrus & & $10,50,24$ & 5.31 & \\
\hline & Left middle cingulate gyrus & 302 & $-4,-24,34$ & 4.98 & 0.035 \\
\hline & Decrease activation & & & & \\
\hline & Left superior occipital gyrus & 20,720 & $-24,-79,42$ & 8.43 & 0.000 \\
\hline & Right superior occipital gyrus & & $30,-76,42$ & 8.24 & \\
\hline & Left inferior temporal gyrus & & $-52,-50,-10$ & 8.17 & \\
\hline & Left inferior triangle frontal gyrus & 2,118 & $52,16,20$ & 6.96 & 0.000 \\
\hline & Right superior frontal gyrus & & $28,6,60$ & 6.39 & \\
\hline & Right insula & & $34,22,12$ & 5.11 & \\
\hline & Left inferior opercular frontal gyrus & 2,590 & $-44,6,28$ & 6.50 & 0.000 \\
\hline & Left middle frontal gyrus & & $-28,0,56$ & 5.18 & \\
\hline \multirow[t]{16}{*}{ PO-NEU } & Increase activation & & & & \\
\hline & Left precuneus & 1,435 & $-10,-56,30$ & 7.65 & 0.000 \\
\hline & Right precuneus & & $10,-58,34$ & 5.90 & \\
\hline & Right medial superior frontal gyrus & 355 & $4,54,26$ & 5.26 & 0.020 \\
\hline & Left medial superior frontal gyrus & & $-8,46,50$ & 4.13 & \\
\hline & Decrease activation & & & & \\
\hline & Left inferior temporal gyrus & 21,714 & $-52,-52,-8$ & 8.28 & 0.000 \\
\hline & Right fusiform gyrus & & $36,-52,-22$ & 7.93 & \\
\hline & Right superior occipital gyrus & & $28,-78,46$ & 7.84 & \\
\hline & Left inferior opercular frontal gyrus & 3,447 & $-40,4,26$ & 8.24 & 0.000 \\
\hline & Left superior frontal gyrus & & $-24,-4,46$ & 6.31 & \\
\hline & Right precentral gyrus & 2,280 & $28,-2,48$ & 7.04 & 0.000 \\
\hline & Right inferior triangle frontal gyrus & & $48,14,22$ & 6.08 & \\
\hline & Right precentral gyrus & & $40,2,28$ & 5.92 & \\
\hline & Left thalamus & 313 & $-18,-28,14$ & 6.05 & 0.030 \\
\hline & Right middle cingulate gyrus & 291 & $8,4,34$ & 5.02 & 0.038 \\
\hline
\end{tabular}

NS-NEU: negative-self minus neutral contrast, NO-NEU: negative-others minus neutral contrast, PS-NEU: positive-self minus neutral contrast, PO-NEU: positive-others minus neutral contrast, MNI: Montreal Neurological Institute, pFWE: probability family wise error 


\begin{tabular}{|c|c|c|c|c|c|}
\hline Task & Brain region & Cluster size & $\begin{array}{c}\text { MNI coordinates } \\
\text { of peak voxel }\end{array}$ & t-value & pFWE corrected \\
\hline \multirow[t]{15}{*}{ NS-NEU } & Increase activation & & & & \\
\hline & Left medial orbit frontal gyrus & 7,430 & $-10,50,-4$ & 10.00 & 0.000 \\
\hline & Left medial superior frontal gyrus & & $-8,52,8$ & 9.54 & \\
\hline & Left anterior cingulate gyrus & & $-10,40,-6$ & 9.44 & \\
\hline & Left precuneus & 4,162 & $-10,-52,34$ & 8.38 & 0.000 \\
\hline & Right middle cingulate gyrus & & $8,-52,34$ & 8.25 & \\
\hline & Left angular gyrus & 377 & $-52,-70,34$ & 8.22 & 0.015 \\
\hline & Decrease activation & & & & \\
\hline & Right insula & 9,840 & $38,22,6$ & 9.74 & 0.000 \\
\hline & Right superior frontal gyrus & & $26,2,68$ & 7.90 & \\
\hline & Right middle frontal gyrus & & $38,36,26$ & 7.62 & \\
\hline & Right angular gyrus & 20,269 & $36,-56,48$ & 8.35 & 0.000 \\
\hline & Left inferior parietal gyrus & & $-28,-64,42$ & 8.13 & \\
\hline & Right hippocampus & 843 & $26,-34,8$ & 5.85 & 0.000 \\
\hline & Right calcarine & & $24,-54,6$ & 5.13 & \\
\hline \multirow[t]{11}{*}{ NO-NEU } & Increase activation & & & & \\
\hline & Left precuneus & 1,223 & $-8,-66,34$ & 6.86 & 0.000 \\
\hline & Right precuneus & & $8,-56,34$ & 6.15 & \\
\hline & Right medial superior frontal gyrus & 1,558 & $8,62,20$ & 6.34 & 0.000 \\
\hline & Left medial superior frontal gyrus & & $-6,60,14$ & 5.82 & \\
\hline & Decrease activation & & & & \\
\hline & Left precentral gyrus & 13,802 & $-46,8,32$ & 10.58 & 0.000 \\
\hline & Left insula & & $-34,16,12$ & 8.75 & \\
\hline & Right inferior triangle frontal gyrus & & $36,24,8$ & 8.61 & \\
\hline & Left calcarine & 43,070 & $4,-90,10$ & 10.36 & 0.000 \\
\hline & Right anterior cingulate gyrus & 424 & $4,6,28$ & 6.21 & 0.009 \\
\hline \multirow[t]{13}{*}{ PS-NEU } & Increase activation & & & & \\
\hline & Right precuneus & 16,498 & $34,-28,56$ & 8.86 & 0.000 \\
\hline & Left precuneus & & $-8,-66,34$ & 8.60 & \\
\hline & Left medial superior frontal gyrus & & $-12,52,0$ & 8.44 & \\
\hline & Decrease activation & & & & \\
\hline & Left inferior parietal gyrus & 28,319 & $-30,-68,40$ & 9.12 & 0.000 \\
\hline & Left superior parietal gyrus & & $-18,-72,58$ & 8.97 & \\
\hline & Right insula & 5,780 & $32,22,-2$ & 8.87 & 0.000 \\
\hline & Right middle frontal gyrus & & $44,40,28$ & 8.19 & \\
\hline & Right opercular inferior frontal gyrus & & $48,18,10$ & 6.87 & \\
\hline & Left superior frontal gyrus & 7,266 & $-26,6,68$ & 8.02 & 0.000 \\
\hline & Left middle frontal gyrus & & $-26,8,58$ & 7.85 & \\
\hline & Left insula & & $-32,16,12$ & 7.45 & \\
\hline \multirow[t]{8}{*}{ PO-NEU } & Increase activation & & & & \\
\hline & Left precuneus & 1,253 & $-10,-66,34$ & 7.07 & 0.000 \\
\hline & Right precuneus & 628 & $34,-26,56$ & 5.73 & 0.001 \\
\hline & Right insula & 640 & $42,-12,8$ & 5.52 & 0.001 \\
\hline & Right medial orbit frontal gyrus & 762 & $8,52,-6$ & 5.41 & 0.000 \\
\hline & Left medial orbit frontal gyrus & & $-8,54,-6$ & 5.24 & \\
\hline & Decrease activation & & & & \\
\hline & Left inferior temporal gyrus & 61,699 & $-50,-52,-8$ & 11.72 & 0.000 \\
\hline
\end{tabular}


Supplementary Table 3. Peak activations during the NS-NEU, NO-NEU, PS-NEU and PO-NEU contrasts in drug-naïve/drug-free patients

\begin{tabular}{|c|c|c|c|c|c|}
\hline Contrast & Brain region & Cluster size & $\begin{array}{c}\text { MNI coordinates } \\
\text { of peak voxel }\end{array}$ & $\mathrm{t}$-value & pFWE corrected \\
\hline \multirow[t]{8}{*}{ NS-NEU } & Patient $>$ control & & & & \\
\hline & Left superior frontal gyrus & 34,761 & $-16,10,66$ & 6.43 & 0.000 \\
\hline & Left inferior triangle frontal gyrus & & $-36,30,2$ & 5.24 & 0.000 \\
\hline & Right cerebelum crus 1 & & $34,-78,-28$ & 5.14 & 0.000 \\
\hline & Patient $<$ control & & & & \\
\hline & Right insula & 6,278 & $36,-16,14$ & 6.12 & 0.001 \\
\hline & Right precentral gyrus & & $42,-32,64$ & 4.97 & 0.001 \\
\hline & Right parahippocampal gyri & & $28,-20,-20$ & 4.75 & 0.001 \\
\hline \multirow[t]{4}{*}{ NO-NEU } & Patient $>$ control & & & & \\
\hline & Left cerebelum crus 2 & 53,168 & $-10,-76,-32$ & 6.37 & 0.000 \\
\hline & Right cerebelum crus 2 & & $12,-80,-42$ & 6.26 & 0.000 \\
\hline & Right anterior cingulate gyrus & & $6,42,28$ & 6.00 & 0.000 \\
\hline \multirow[t]{7}{*}{ PS-NEU } & Patient $>$ control & & & & \\
\hline & Right superior frontal gyrus & 6,244 & $18,16,52$ & 4.75 & 0.001 \\
\hline & Right opercular inferior frontal gyrus & & $46,8,22$ & 4.38 & 0.001 \\
\hline & Right medial superior frontal gyrus & & $6,40,42$ & 3.72 & 0.001 \\
\hline & Left precuneus & 8,008 & $-10,-76,48$ & 4.72 & 0.000 \\
\hline & Right middle temporal gyrus & 14,364 & $60-56,12$ & 4.38 & 0.000 \\
\hline & Right middle temporal gyrus & & $40-64,16$ & 4.34 & 0.000 \\
\hline \multirow[t]{4}{*}{ PO-NEU } & Patient $>$ control & & & & \\
\hline & Right cerebellum 6 & $1,002,130$ & $16,-74,-26$ & 8.60 & 0.000 \\
\hline & Left cerebellum 6 & & $-12,-72-26$ & 7.68 & 0.000 \\
\hline & Right cerebellum crus 1 & & $34,-80,-26$ & 6.50 & 0.000 \\
\hline
\end{tabular}

NS-NEU: negative-self minus neutral contrast, NO-NEU: negative-others minus neutral contrast, PS-NEU: positive-self minus neutral contrast, PO-NEU: positive-others minus neutral contrast, MNI: Montreal Neurological Institute, pFWE: probability family wise error 